\title{
The challenges facing systemic approaches in biology: an interview with Kunihiko Kaneko
}

\section{Kunihiko Kaneko*}

Research Center for Complex Systems Biology, University of Tokyo, Tokyo, Japan

Edited by:

Kumar Selvarajoo, Keio University, Japan

Reviewed by:

Kumar Selvarajoo, Keio University, Japan

Masa Tsuchiya, Keio University, Japan

${ }^{*}$ Correspondence:

Kunihiko Kaneko, Research Center for

Complex Systems Biology, University

of Tokyo, Meguro, Komaba, Tokyo

153-8902, Japan.

e-mail: kaneko@complex.c.u-tokyo.

ac.jp
Interviewed by Kumar Selvarajoo and MasaTsuchiya at Institute for Advanced Biosciences, Keio University, Japan, I discuss my approach to biology, what I call complex systems biology. The approach is constructive in nature, and is based on dynamical systems theory and statistical physics. It is intended to understand universal characteristics of life systems; generic adaptation under noise, differentiation from stem cells in interacting cells, robustness and plasticity in evolution, and so forth. Current status and future directions in systems biology in Japan are also discussed.

\section{Keywords: plasticity, adaptation, robustness, stem cell, noise}

\section{1: PROFESSOR KANEKO, CAN YOU INTRODUCE YOURSELF AND YOUR RESEARCH?}

I have been a physicist, and although I am more and more involved in biology now, I think my approach is quite a physicist-type. I started my graduate studies in the field of non-equilibrium phenomena in terms of stochastic process, and then worked on chaos, a deterministic dynamics that produce irregular, "unpredictable" behavior (Kaneko, 1986). Then, my study shifted to chaos in space and time, having many degrees of freedom. I introduced the "coupled map model," which proved to be a powerful tool that allows one to study the properties of dynamical systems with many degrees of freedom. Key concepts that derived from it such as collective dynamics and chaotic itinerancy have had impacts on a variety of fields, ranging from turbulence in fluid dynamics to neural activities in the brain. A book "Complex Systems: Chaos and Beyond" (Kaneko and Tsuda, 2000) that I wrote together with Ichiro Tsuda, to a certain extent I believe, mediated the "Japanese own taste for complex systems" (as reviewed in Nature; Shlesinger, 2001) to scientists abroad.

Based on these studies and concepts, I proposed "Complex Systems Biology" at around 1994, to unveil universal properties in a life system. It is not easy to judge if some features in the present organism are chance or necessity, as they are shaped as a result of one-time evolution in this Earth. It is not so sure if the features appeared again when the tape of life were replayed. To unveil universal, essential features in life, it is then ideal to construct some basic process of life (such as reproduction, adaptation, differentiation, and so forth) and examine generic features therein. This is a constructive approach Tetsuya Yomo at Osaka University and I proposed in mid 1990s. The earlier works including collaborated studies with experimental biologists Yomo and Makoto Asashima at University of Tokyo are described in the book "Life: An Introduction to Complex Systems" (Kaneko, 2006). During these years I have served as a director of Center-of-Excellence Project "Search for the Logic of Life as a Complex System" (1999-2004) and the ERATO project "Kaneko Complex Systems Biology" (20042010), and am a head of Center for Complex Systems Biology at University of Tokyo.

\section{2: WHEN AND HOW DID YOU BECOME INTERESTED IN BIOLOGICAL RESEARCH?}

From the beginning of graduate studies at 1979 I was interested in "what life is." My intention was to understand its universal characteristics, and what distinguishes life from non-living matter. So I hoped to understand what life is, theoretically, in terms of physics. At that time, Prigogine's "dissipative structure" was popular among statistical physicists, in which the ultimate goal would be to understand life as a spatiotemporal pattern possible in far-from-equilibrium state. However, there was a large gap between such studies in physic-chemical systems and life systems. So I could not start biological research seriously until 1992, when I first met Tetsuya Yomo at a meeting organized by Professor Yuzuru Fushimi. I was then working on "globally coupled maps," in which simple identical dynamic elements interact with every other in the same way. I found that even though these elements are identical, their behaviors start to differ from each other with time and then form a few groups within which the behaviors are identical but the behaviors of elements belonging to different groups are distinct (Kaneko, 1990). As this "differentiation" occurs across elements sharing the identical "rule," I had thought that this might be similar with the differentiation of cells that share the identical gene. This similarity, however, had remained to be at "metaphorical" level. At that time Tetsuya discovered that bacteria sharing the same gene differentiated into active and inactive types, even in a well mixture culture (Ko et al., 1994), and was seeking mechanistic interpretation for it. I explained to him how my simple elements of coupled maps differentiated, with a remark on "why not bacteria that have more complex dynamics within?”. So, we started collaboration. This 
study on prototypical cell differentiation was also theoretically interesting, as the number of elements ("cells") change through division and death, which aspect had not been studied in physics before. Since then, we have continued collaboration to unveil basic logic in cell reproduction, heredity, adaptation, development, and evolution.

\section{3: CAN YOU SHARE INITIAL EXPERIENCES WORKING WITH BIOLOGISTS?}

I think I was quite lucky with this initial experience. Tetsuya Yomo has always been interested in general (universal) aspects, and does not like such explanation that life system is special and finely designed through evolution. We always try to understand a characteristic property of life system as a general consequence of a system that grows autonomously. So we have had a common picture, and have enjoyed the collaboration. This, however, would be atypical experience, since his way of thinking is far away from traditional biologists. By the way, my way of thinking will be probably out of standard physicists'. In the beginning we misunderstood the way of thinking of each other as that of a typical biologist and physicist, but that was wrong. Biologists generally are not so much interested in universal properties or minimal models. For example, they often ask me "why do not you add this and that processes to make my model more realistic," while I make effort to reduce the complicatedness in the model. In physics, there are several abstract models that do not fit the details of the nature but are essential to understand universal features and unveil general laws, say Carnot cycle, ideal gas, Ising model for phase transition, and so forth. In this sense, I believe that learning how physics has succeeded in extracting universal laws in nature is essential to establish theories for a life system.

\section{4: PLEASE HIGHLIGHT YOUR MAJOR FINDINGS IN A SIMPLE WAY}

Our "complex systems biology" is distinguishable from the socalled "systems biology" developed in recent years (Kaneko, 2006). By the word "complex," we do not mean "complicated." Systems that consist of many elements, e.g., molecules within a cell or cells within an organism exhibit homeostasis. Such systems should be constrained so that consistency between each element and the whole system is maintained, while keeping the reproduction of both elements and the system. Indeed, we found that one can derive widely applicable rules in the dynamics of gene expression and cellular organizations during adaptation to environment, reproduction, cell differentiation, and evolution. To list a few examples:

(i) Cell reproduction: in a cell that reproduces itself, all molecules are replicated keeping the composition to some degree. We found that this constraint on consistent reproduction leads to universal law on statistical distribution of abundances of each protein, as well as their fluctuations around their average values across cells (Furusawa and Kaneko, 2003). This law will give a criterion for a steady state of cells, while the fluctuations over cells are important in adaptation and evolution, as will be discussed below. (ii) Emergence of "natural adaptation," for any growing cells under stochastic gene expression, even without the use of specific signal transduction network (see more discussion in Q7; Kashiwagi et al., 2006; Furusawa and Kaneko, 2008). This may provide a theoretical basis flexibility of cells as well as multicellular organisms to adapt a huge variety of environmental conditions.

(iii) Differentiation from a stem cell to committed cells, as a general consequence of interacting cells whose gene expression (abundances of some proteins) exhibits temporal oscillation (Furusawa and Kaneko, 1998, 2001; Suzuki et al., 2011). Here this oscillation is not completely periodic, but involves some irregularity. In fact, this irregular oscillation can show some instability in the expression state to switch to a different state upon cell-cell interaction. Differentiated (committed) cells, then, appear which lose such oscillation and stemness (see also in Q7, and the introduction of Kaneko, 2011b for more description).

(iv) General proportionality between variances of phenotype due to genetic change and due to noise. In other words, the variability of phenotype by genetic change (mutation) is correlated with that by stochastic gene expression, or put differently, the phenotype that has higher fluctuation without genetic change has higher evolution speed (that is proportional to variability by genetic change). In this way, we can characterize the degree of evolvability. According to our theory, this proportionality is also formulated in terms of robustness, i.e., the robustness to noise in gene expression leads to robustness to genetic change through the evolution (Sato et al., 2003; Kaneko and Furusawa, 2006; Kaneko, 2007).

\section{5: WHY DO YOU FEEL STRONGLY FOR THE EXISTENCE OF GOVERNING RULES IN LIVING SYSTEMS?}

Of course there is no logical reasoning to demonstrate the existence of universal laws in a living system, represented by few degrees of variables. However, trained biologists have intuition on activity, plasticity, and stability that make things "lively." They somehow characterize a "liveliness" by compressing detailed information in such life system. Probably, they have some concept on liveliness or biological activity, which does not necessarily require a huge number of parameters, but is represented by a few. This suggests that there exists some underlying logic in life that is represented in terms of few variables. So far we do not know such variables explicitly, though. This situation somewhat gives me an impression that we are in the time just before "thermodynamics" was established; We had sense on "hot" or "cold" but had not yet established the quantitative concept of temperature. Later we reached the concept of temperature and entropy, from which we reached the universal laws in thermodynamics. We have a sense on biological activity, plasticity, and robustness, but have not reached a proper mathematical formulation yet. Anyway, living state is a very common form of things (at least in this Earth and probably in the Universe I hope), and as a genuine physicist, it is natural to expect the existence of universal laws that govern such state. 


\section{6: NOISE IN BIOLOGY HAS RECEIVED SIGNIFICANT ATTENTION IN RECENT YEARS. WHAT ARE YOUR EXPERIENCES ON THIS?}

At the end of 1990s when we proposed "isologous-diversification" theory for cell differentiation, one key issue was that the amplification of noise then leads to robust cell distribution through cell-cell interaction (Kaneko and Yomo, 1994, 1999). Our view was summarized as "noise-amplification leads to noisetolerant cell society," as small variation in protein concentration is amplified in the irregular oscillation I mentioned earlier in Q5.

By looking at the data on the distribution of fluorescence of proteins in bacteria cells obtained by flow cytometry experiments by Yomo's group at around 2000, we soon recognized that the cellto-cell variance is quite large, and furthermore the distribution of fluorescence (or protein concentration) does not obey Gaussian (normal) distribution, but the logarithm of them does. We have shown that this is a necessity outcome of a multiplicative stochastic process - this is common in catalytic reactions, as the rate equation of chemical reaction, in general, has a multiplicative form between substrate and catalyst and their concentrations fluctuate (Furusawa et al., 2005). The Gaussian distribution of logarithm of the concentration that cell-to-cell variation by noise sometimes ranges to the order of magnitude.

Then we were more interested in relevance of such fluctuations to adaptation and evolution. During evolution, individuals with higher fitness are selected. Developmental dynamics that give rise to such individuals are continuously bombarded by noise in signal transduction, transcription, or translation. Since this generally perturbs the optimal phenotype, most studies focus on how developmental systems reduce or eliminate such disturbances. However, considering the recent observations of large noise in gene expression, it is natural to ask whether there is any positive role that noise plays in the biological organization and evolution. By combination of statistical physics based theory and evolutionary experiments in the lab, we have demonstrated that there is a positive correlation between noise and the rate of evolution. In other words, developmental robustness to noise facilitates robustness against mutation [see also Q4 (iv)]. This adds a new dimension to the classic problem of nature versus nurture as it suggests a strong relationship between phenotypic variation by mutation and that by developmental noise (Sato et al., 2003; Kaneko, 2007, 2011a).

As for adaptation at a single-cell level, Kashiwagi, Urabe, Yomo, Furusawa, and myself proposed a generic process for it, by noting that stability of growing cells against intrinsic noise is higher than non-growing cells, and there is general tendency that a cell switched to a state with higher growth by a noise in gene expression (Kashiwagi et al., 2006; Furusawa and Kaneko, 2008). This leads to "natural adaptation" of any cells even without the use of specific signal transduction network. It will be relevant to understand why bacteria, for example, can adapt to a huger variety of different environments which they probably have not met before.

\section{7: CAN YOUR FINDINGS BE VALIDATED EXPERIMENTALLY? IF NOT, WHY AND WILL THIS CHANGE IN THE FUTURE?}

When Chikara Furusawa and I proposed that irregular oscillation in gene expression provides stemness more at 1998, many did not believe in the existence of such oscillation. This is because they measured the average of gene expressions over many cells at that time. As long as the oscillation is not synchronized, oscillation in gene expression, if it existed, would be averaged out and could not be observed. Now, one can measure a protein expression in a single-cell by imaging techniques. Indeed 2 years ago, Kobayashi et al., 2009; Kageyama's group) found the oscillation in $\mathrm{HeS}$ protein expression in embryonic stem cell. To our great pleasure, this oscillation disappeared in differentiated cells, as is consistent with our theory.

The studies on adaptation and evolution I mentioned started from experiments, and in this sense, the theory is formulated to be consistent with experimental findings. Then, the theory, in turn, can predict something more, which should be confirmed experimentally. Besides such confirmation, the experiments later challenge theorists with new findings. Ideally, theory and experiments progress hand in hand, in a form of expanding spiral.

For example, the evolution study started from the analysis of the experiment in Yomo's group, and thus in the beginning we proposed a theory to be consistent with experiments, i.e., the proportionality between evolution speed and isogenic fluctuation of phenotype by noise. Then our theory and simulations make new predictions, the proportionality between this isogenic fluctuation and the genetic variance that is the fluctuation due to genetic variation. Now it is a turn to check this relationship experimentally, which is ongoing, and I am looking forward to hearing a positive report soon.

As mentioned, study of "natural adaptation" stemmed from an experiment by Kashiwagi et al. (2006). By embedding an artificial gene network into bacteria, we demonstrated that $\mathrm{E}$. coli are able to adapt to an optimal-growth state without the need for a specific induction mechanism. The accordingly proposed theory of ours is general, so that now it is a turn to carry out an experiment to demonstrate that this natural adaptation indeed works in natural conditions or in a higher organism.

\section{8: DO YOU THINK JAPAN WILL OPEN DOORS AND INVITE INTERNATIONAL SCIENTISTS TO DO SYSTEMS BIOLOGY RESEARCH IN THE FUTURE?}

Generally, the answer is yes. Especially, in research institutes, the doors have already been opened, and this will be further accelerated. As for universities, we have to decide if we start to give lectures regularly in English. Considering the decrease in population in younger ages, we need to accept immigration more, and at some point we probably have to decide it. To some degree this will be good, but I also have some concern. Giving lectures and thinking in one' own language in science may be important to cultivate creativity. As the world will be "Americanized," the originality in each culture may be declined, which may also suppress developing original ideas in science. In fact, the originality in scientific activity was quite high when Japan was more isolated, to give some examples, Yukawa's meson theory, linear response theory in statistical physics, and so forth. As the world is homogenized, the frequency of original breakthrough in science seems to be declined. Of course, whether we can expect true breakthrough in the systems biology (as we experienced in the emergence of thermodynamics, quantum mechanics, general relativity, Darwinian evolution theory, and so forth) is another question, though. 
I may be one of the minority who expects that real theoretical breakthrough will take place in biology, and for it, I believe that thinking differently under an appropriate level of isolation is important.

\section{9: WHAT ARE YOUR FUTURE ASPIRATIONS?}

(1) Set up a general theory on what life is and fill a gap between just a set of chemical reactions and a life system, while waiting for a discovery of life in the outer space.

(2) Establish an appropriate phenomenological theory for universal properties on adaptation, development, and evolution, as mentioned in the answer to Q5. Once this is done, we can

\section{REFERENCES}

Furusawa, C., and Kaneko, K. (1998). Emergence of rules in cell society: differentiation, hierarchy, and stability. Bull. Math. Biol. 60, 659-687.

Furusawa, C., and Kaneko, K. (2001). Theory of robustness of irreversible differentiation in a stem cell system: chaos hypothesis. J. Theor. Biol. 209, 395-416.

Furusawa, C., and Kaneko, K. (2003). Zipf's law in gene expression. Phys. Rev. Lett. 90, 088102

Furusawa, C., and Kaneko, K. (2008). A generic mechanism for adaptive growth rate regulation. PLoS Comput. Biol. 4, e3. doi:10.1371/journal.pcbi.0040003

Furusawa, C., and Suzuki, T., Kashiwagi, A, Yomo, T., and Kaneko, K. (2005). Ubiquity of log-normal distributions in intra-cellular reaction dynamics. Biophysics 1, 25.

Kaneko, K. (1986). Collapse of Tori and Genesis of Chaos in Dissipative Systems. Singapore: World Scientific Publishing.

Kaneko, K. (1990). Clustering, coding, switching, hierarchical ordering, and control in network of chaotic elements. Physica 41D, 137-172.
Kaneko, K. (2006). Life: An Introduction to Complex Systems Biology. Heidelberg: Springer.

Kaneko, K. (2007). Evolution of robustness to noise and mutation in gene expression dynamics. PLoS ONE 2, e434. doi:10.1371/journal.pone.0000434

Kaneko, K. (2011a). Proportionality between variances in gene expression induced by noise and mutation: consequence of evolutionary robustness. BMC Evol. Biol. 11, 27. doi:10.1186/1471-2148-11-27

Kaneko, K. (2011b). Characterization of stem cells and cancer cells on the basis of gene expression profile stability, plasticity and robustness. Bioessays 33, 403-413.

Kaneko, K., and Furusawa, C. (2006). An evolutionary relationship between genetic variation, and phenotypic fluctuation. J. Theo. Biol. 240, 78-86.

Kaneko, K., and Tsuda, I. (2000). Complex Systems: Chaos and Beyond. Heidelberg: Springer.

Kaneko, K., and Yomo, T. (1994). Cell division, differentiation, and dynamic clustering. Physica 75D, 89-102.

also understand the condition to recover multipotency in a cell, and characterize a cancer cell.

\section{0: YOUR ADVICE TO NON-BIOLOGISTS WHO CONSIDER APPLYING THEIR SKILLS IN BIOLOGY}

I have been interested in what life is, and to answer it I need first to know universal features in non-life system, for which physics is important. So far, I think mathematics is useful to understand nature reasonably, and as for the application of mathematics for natural science, physics has been most successful. So, I recommend to study seriously what life is, in terms of physics and mathematics, but this may be my biased viewpoint.

Kaneko, K., and Yomo, T. (1999). Isologous diversification for robust development of cell society. J. Theor. Biol. 199, 243-256.

Kashiwagi, A., Urabe, I., Kaneko, K., and Yomo, T. (2006), Adaptive response of a mutually inhibitory gene network to environmental changes by attractor selection. PLOS ONE 1, e49. doi:10.1371/journal.pone. 0000049

Ko, E., Urabe, I., and Yomo, T. (1994). Dynamic clustering of bacterial population. Physica 75D, 81.

Kobayashi, T., Kobayashi, T., Mizuno, H., Imayoshi, I., Furusawa, C., Shirahige, K., and Kageyama, R. (2009). The cyclic gene Hesl contributes to diverse differentiation responses of embryonic stem cells. Genes Dev. 23, 1870-1875.

Sato, K., Ito, Y., Yomo, T., and Kaneko, K. (2003). On the Relation between fluctuation and response in biological systems. Proc. Natl. Acad. Sci. U.S.A. 100, 14086-14090.

Shlesinger, M. F. (2001). When space and time conspire. Nature 410, 1028-1029.

Suzuki, N., Furusawa, C., and Kaneko, K. (2011). Oscillatory protein expression dynamics endows stem cells with robust differentiation potential. PLoS ONE 6, e27232. doi:10.1371/journal.pone.0027232

Conflict of Interest Statement: The author declares that the research was conducted in the absence of any commercial or financial relationships that could be construed as a potential conflict of interest.

Received: 16 November 2011; accepted: 18 November 2011; published online: 05 December 2011.

Citation: Kaneko K (2011) The challenges facing systemic approaches in biology: an interview with Kunihiko Kaneko. Front. Physio. 2:93. doi: 10.3389/fphys.2011.00093

This article was submitted to Frontiers in Systems Physiology, a specialty of Frontiers in Physiology.

Copyright (c) 2011 Kaneko. This is an open-access article distributed under the terms of the Creative Commons Attribution Non Commercial License, which permits non-commercial use, distribution, and reproduction in other forums, provided the original authors and source are credited. 